\title{
A cost-utility analysis in patients receiving enteral tube feeding at home and in nursing homes
}

\author{
R. J. Stratton and M. Elia on behalf of the British Artificial Nutrition Survey (BANS) \\ Institute of Human Nutrition, University of Southampton, Southampton SO16 6YD, UK
}

Decisions about the distribution of limited financial resources in the NHS and other health care systems can be aided by a cost-utility analysis. This study aimed to undertake a cost-utility analysis to establish the cost per quality adjusted life years (cost/QALY) of ETF in patients with cerebrovascular accident (CVA), one of the largest patient groups registered with the British Artificial Nutrition Survey (BANS).

Data were obtained from an audit carried out by BANS on patients with a CVA who started enteral tube feeding (ETF), either in their own home $(n$ 2888) or in nursing homes $(n$ 7007). Clinical status of patients (including mortality, time spent in hospital etc.) was assessed every 3 months from the start of ETF for up to 3 years. Quality of life was established using the EuroQol visual analogue scale which scores quality of life from 0 (worst possible quality of life) to 1 (best possible quality of life). This quality of life questionnaire, which was sent to a randomly selected sample of patients via reporters, was completed for twenty-five patients with a CVA, living in their own home (age $72 \pm 9$ years) or in nursing homes (age $76 \pm 11$ years). QALY were calculated over 3 years using mortality and mean quality of life score. The relevant costs (tube insertion, feed and ancillaries (assumed no delivery charges for NHS), $4 \mathrm{~d}$ spent in hospital training, home visits from NHS professionals (including dietitian, GP, district nurse, other health professionals), monitoring (blood tests), hospital readmission (7.46 d/year)) were calculated and the cost/QALY estimated. For patients in nursing homes, the medical costs, which are fully met by the NHS, were included. As the contribution made by the state to non-medical costs can vary depending on the individual's condition and personal resources, in this analysis it was assumed to vary from $0 \%$ to $100 \%$. It was also assumed that no training would occur prior to hospital discharge for nursing home patients and that there would be no district nursing costs. The number of days nursing home patients spent in hospital was less $(3.20 \mathrm{~d})$ than those at home. For both groups it was assumed that death would occur immediately without ETF.

\begin{tabular}{lcc}
\hline & Home $(n$ 10) & Nursing home $(n$ 15) \\
\hline Quality of life EuroQol score $(0-1)^{*}$ & $0.47 \pm 0.28$ & $0.47 \pm 0.25$ \\
Cost/QALY & $£ 12817$ & $£ 10303-£ 68065 \dagger$ \\
\hline There was no significant difference between groups and no significant trend since the start of ETF. \\
$\dagger$ Figures indicate cost/QALY when $0 \%$ to $100 \%$ of non-medical costs for nursing home patients are paid by state.
\end{tabular}

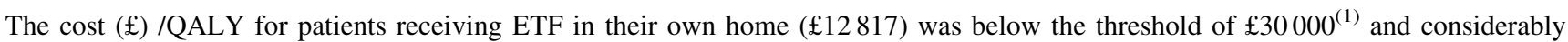
lower than if the patients remained in an NHS hospital ( $£ 171727 /$ QALY, assuming the same outcome). In nursing home patients, the cost/ QALY varied considerably depending on the contributions made by the state to non-medical costs (Table).

This data suggests that currently ETF is much more cost-effective for the health care system, when used in the home or in nursing homes (where the non-medical costs are paid privately) than in NHS hospitals. The cost-effectiveness of ETF is less in a nursing home when the state pays all non-medical costs, raising issues of economic and ethical concern.

1. Raftery J. BMJ 2006, 332, 1266-1268. 